\title{
Nonlinear-Elastic Orthotropic Material Modeling of an Epoxy-Based Polymer for Predicting the Material Behavior of Transversely Loaded Fiber-Reinforced Composites
}

\author{
Caroline Lüders ${ }^{1,2}$ (1) \\ 1 Institute of Adaptronics and Function Integration, Technische Universität Braunschweig, Langer Kamp 6, \\ 38106 Braunschweig, Germany; c.lueders@tu-braunschweig.de \\ 2 Institute of Composite Structures, German Aerospace Center, Lilienthalplatz 7, \\ 38108 Braunschweig, Germany
}

Received: 6 April 2020; Accepted: 30 April 2020; Published: 2 May 2020

check for updates

\begin{abstract}
Micromechanical analyses of transversely loaded fiber-reinforced composites are conducted to gain a better understanding of the damage behavior and to predict the composite behavior from known parameters of the fibers and the matrix. Currently, purely elastic material models for the epoxy-based polymeric matrix do not capture the nonlinearity and the tension/compression-asymmetry of the resin's material behavior. In the present contribution, a purely elastic material model is presented that captures these effects. To this end, a nonlinear-elastic orthotropic material modeling is proposed. Using this matrix material model, finite element-based simulations are performed to predict the composite behavior under transverse tension, transverse compression and shear. Therefore, the composite's cross-section is modeled by a representative volume element. To evaluate the matrix modeling approach, the simulation results are compared to experimental data and the prediction error is computed. Furthermore, the accuracy of the prediction is compared to that of selected literature models. Compared to both experimental and literature data, the proposed modeling approach gives a good prediction of the composite behavior under matrix-dominated load cases.
\end{abstract}

Keywords: fiber-reinforced plastics; micromechanics; material modeling; polymeric matrix; elasticity

\section{Introduction}

Fiber-reinforced plastics (FRP) provide excellent mass specific stiffness and strength and are therefore used in a wide range of industrial applications, e.g., in aeronautic, space, and automotive sectors. Due to their inhomogeneous and anisotropic nature, the damage behavior of FRP is complex and requires different modeling strategies compared to metals. Damage and failure in FRP is a multi-scale phenomenon: In a laminate made of several unidirectional (UD) plies with different fiber orientations, matrix damage initiates in the transversally loaded plies [1]. This matrix cracking can be traced back to the failure of the fiber-matrix-interface and a resulting fiber-matrix-debonding. When increasing the load, the debonded areas coalesce to form a through-ply matrix crack [1]. The properties of the fiber-matrix-interface, tailored by the fiber's surface treatment, may have a high impact on the stress transfer between fiber and matrix and on the damage initiation, as concluded in the review by Jones [2]. This leads to the conclusion that the interface influences the effective properties of the composite (e.g., [3,4]).

As the micromechanical damage mechanism in transversally loaded UD plies is a key aspect of the damage of laminates, researchers have focused on the modeling of these phenomena in recent 
years (e.g., [5-16]). While having insight into the micro-scale damage mechanisms, micromechanical modeling is used for predicting the composite stiffnesses and strengths of FRP based on the known material behavior of the fibers and the matrix. Different homogenization techniques are available for the micromechanical modeling of heterogeneous materials and to obtain an effective response influenced by the specific microstructure. Micromechanical homogenization techniques and its application to composite materials are reviewed by [17]. The application of mathematical homogenization is discussed in [18] and the mathematical asymptotic homogenization technique is applied to FRP by [19]. Most approaches to model the progressive damage behavior on the micro-scale are based on numerical homogenization that use the finite element method (FEM) and the representative volume element (RVE) approach.The more general numerical homogenization allows the application to every material model without the need to re-derive the homogenization equations for each material model and, therefore, is also used within the presented study.

The current micromechanical simulations (e.g., [5-16]) yield the highest stresses at the fiber-matrix-interface. Consequently, the damage initiates at these interfaces. Simulations by Asp [6] reveal that the exact position of the damage initiation regarding the fiber circumference depends on the fiber-to-fiber-distance which is related to the fiber volume fraction and the local fiber distribution [20]. For transversally loaded FRP the matrix behavior highly influences the global composite response. Thus, one challenging aspect of the micromechanical simulation is the modeling of the constitutive and damage behavior of the polymeric matrix. The behavior of polymers is characterized by nonlinearity and a different behavior in tension, compression and shear (e.g., [21]). Assuming the matrix behavior to be linear-elastic and isotropic yields reasonably good prediction results for the micro-scale damage and the effective response of UD composites under transverse tension load [5,6,14,22-24]. Composites tend to behave more nonlinearly when loaded under transverse compression and shear. Additionally, the absolute transverse stresses differ depending on whether the composite is subjected to a tensile or compressive loading. To predict the composite behavior, it thus seems relevant to incorporate both the nonlinearity as well as the tension/compression-asymmetry of the pure resin. For modeling these material characteristics, plasticity [7-11,25,26], visco-elasticity [15] and visco-plasticity $[12,13,27,28]$ are used in recent micromechanical simulations of FRP. In addition to the plastic deformations $[29,30]$, nonlinear-elastic and viscous effects also contribute to the stress-strain behavior of polymeric resins [15,28,29]. Haupt [31] provides a schematic illustration of the effects of (non)linear elasticity, plasticity and rate-dependency on the stress-strain behavior. It is also illustrated that nonlinearity can also arise from (nonlinear) elasticity and is not necessarily a result of plasticity. Haupt [31] suggests an experimental program to differentiate the named effects. In addition to the monotonic tests, this includes several additional test types like intermediate relaxation tests. As the proposed test program is very costly, only Kästner [28] and Krause [15] characterize the resin in such a way that the various material effects-(non)linear elasticity, plasticity, rate-dependent behavior - can be differentiated. In most cases, only monotonic tests are conducted and the nonlinearity observed in the stress-strain-curves of the pure resin material is attributed completely to plastic effects and is modeled with pressure-dependent elasto-plastic material models. As the different material effects cannot be differentiated from the monotonic tests alone [31], the assumption that the nonlinearity of the resin is purely elastic in nature is just as valid as the assumption that the nonlinearity is entirely due to plastic effects.

Plasticity models are numerically more complex and expensive than elastic models. Additionally to the global iteration steps needed for simulating nonlinear material behavior, extra iterations are needed on an integration point basis when using common plastic material models.Therefore, a pure elastic constitutive modeling can offer advantages concerning computation time and numerical convergence.

Up to now, the nonlinearity and the tension/compression-asymmetry of the constitutive resin behavior are not captured by the purely elastic models that are used for predicting the behavior of UD composites $[5,6,14,22-24]$. In the present contribution a purely elastic model is presented that 
captures the nonlinearity and the tension/compression-asymmetry by a strain-depended and orthotropic constitutive formulation. To assess the predictive capability of this modeling approach, micromechanical FEM simulations of UD composites with focus on matrix-dominated load cases are conducted. The simulation results are compared to experimental data and the prediction error of the micromechanical simulation is compared to that of other models from the literature. The investigation is performed using a carbon fiber-reinforced plastic (CFRP) made of IM7-fiber and an epoxy resin.

\section{Experimental Material Characterization}

Material models must be calibrated with appropriate material data. Additionally, simulation results must be validated against experimental data in order to evaluate the model's prediction capability. This section delivers information about the determination of the experimental calibration and validation data.

\subsection{Materials}

As the micromechanical modeling is based on the composite constituents, material tests on the fiber and the matrix are needed for the model calibration. Elastic and strength properties of the IM7 fiber are given in [32]. To experimentally characterize the epoxy resin, monotonic quasi-static tests are conducted on pure resin specimens. One aim of the micromechanical simulation is to predict the effective constitutive behavior of composites subjected to different loading conditions. Thus, data from monotonic quasi-static mechanical tests on UD composite specimens is needed for the validation. The composite material considered is made of the IM7 fiber and the same epoxy matrix used for the pure resin tests. The fiber volume fraction (FVF) is $62 \%$.

\subsection{Processing Methods}

For the pure resin tests, flat and cylindrical specimens are manufactured, Figure 1.

For the manufacturing of the flat specimens, plates are cast and the specimens are milled from these plates after curing. The cylindrical specimens are manufactured much similarly, also using a casting process. After curing, the specimens are cut to their final length. The curing temperature for both the plates and the cylinders, is $150^{\circ} \mathrm{C}$.

For the UD composite tests, flat rectangular composite specimens are manufactured. To this end, an autoclave vacuum infusion process is used. Additionally, tube specimens are manufactured by a filament winding process with the fiber direction pointing in hoop direction. For both composite specimen types, the curing temperature is $150^{\circ} \mathrm{C}$. The clamping areas of the rectangular composite specimens are reinforced with end tabs made of glass fiber-reinforced plastic (GFRP).

\subsection{Characterization}

For the material characterization, a standard servo-hydraulic test rig by Zwick is used. For the pure resin specimens, uniaxial tension tests are performed according to DIN EN ISO 527-2 using dog-bone shaped specimens according to EN ISO 3167, Figure 1a. The compression tests are orientated to ISO 604, but use cylindrical specimens with a diameter of $16 \mathrm{~mm}$ and a height of $20 \mathrm{~mm}$, Figure $1 \mathrm{~b}$. For applying shear loading, the V-notched beam test according to ASTM D5379 05 is used. The specimen geometry is shown in Figure 1c. The force signal is recorded by appropriate load cells. A biaxial extensometer is used for the strain measurements during the tensile tests. As both the longitudinal as well as the transverse strains are measured, the Poisson's ratio can be determined. During the shear tests, optical digital image correlation (DIC) is used to measure the strains. During the compression tests, strain gauges as well as the displacement signal of the compression plate are used to determine the specimen's strain. The latter measurement incorporates a correction with respect to the machine compliance. 


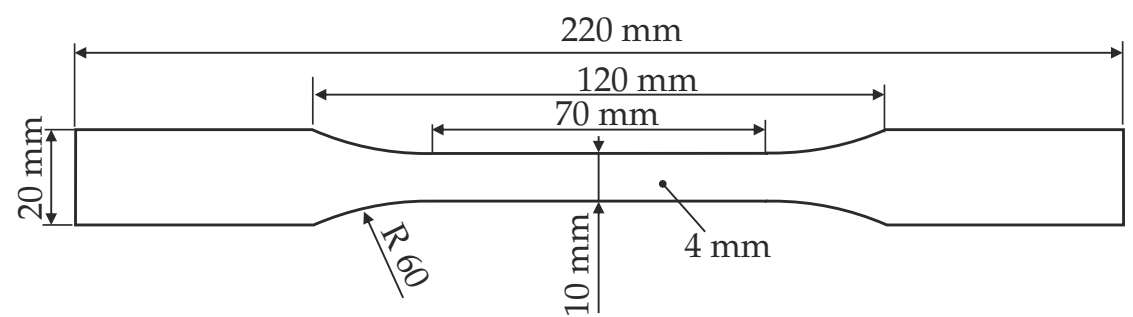

(a)

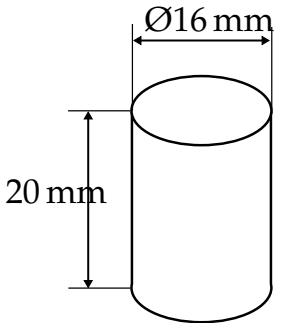

(b)

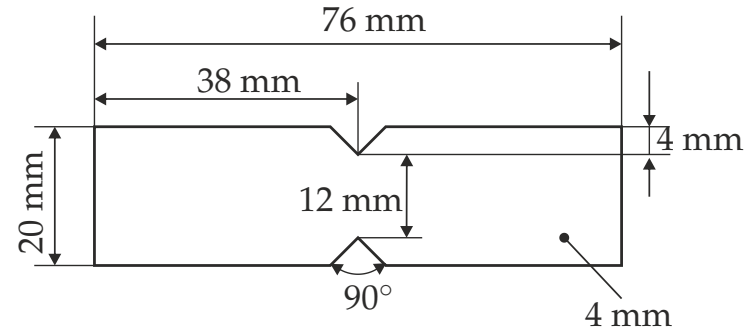

(c)

Figure 1. Specimen geometry for pure resin tests: (a) tension, (b) compression and (c) shear.

For the compression tests, Teflon foil and silicone paste is applied between the specimens and the surfaces of the compression plates to reduce friction. This reduces the obstruction of the transverse contraction of the specimen during compression tests and ensures a uniaxial compressive stress state within the specimens.

The number of available specimens is six for tensile and compression tests and five for the shear test. The stress-strain-relation obtained from the uniaxial tension and compression tests of the pure resin are shown in Figure 2. The according shear test results are shown in Figure 3.

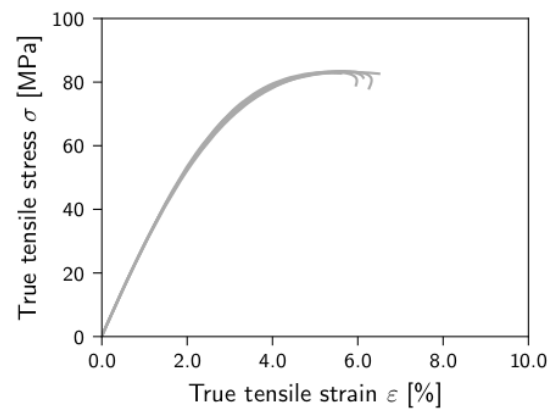

(a)

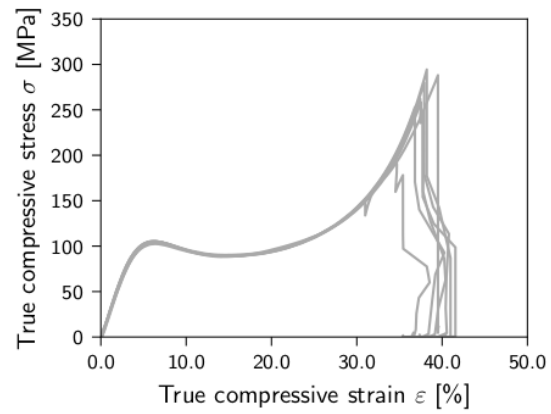

(b)

Figure 2. Experimentally observed stress-strain behavior of the epoxy resin under uniaxial normal loading: (a) tension and (b) compression.

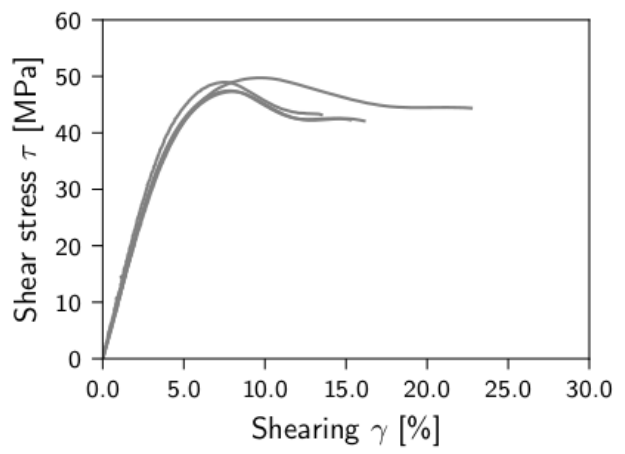

Figure 3. Experimentally observed stress-strain behavior of the epoxy resin under shear loading. 
The technical stresses and strains recorded during the test, are converted into true stresses and true strains [33], p. 12f. For this, the Poisson's ratio is needed which is determined to be $v=0.395$.

As discussed in the introduction, the investigated epoxy resin behaves significantly nonlinear for all load cases and is asymmetric with regards to tensile and compressive loading.

As the present study focusses on transversely loaded composites, the validation database is created with the results of transverse compression, transverse tension and 12-shear composite tests. For transverse tension and transverse compression tests, flat rectangular specimens are used. The shear tests are performed by the Industrieanlagen-Betriebsgesellschaft $\mathrm{mbH}$ (IABG) using tube specimens according to [34] with the fibers pointing in hoop direction. Table 1 gives the related standards or references, the number and the size of the test specimens as well as the length of the clamping area. The flat specimens are equipped with GFRP end tabs in this clamping area. For the strain measurement, strain gauges or biaxial extensometer are used. The applied force is recorded by an appropriate load cell.

Table 1. Mechanical test types and specimen dimensions for UD composite experiments.

\begin{tabular}{lcccc}
\hline Test Type & $\begin{array}{c}\text { Number of } \\
\text { Specimens }\end{array}$ & Standard/Reference & $\begin{array}{c}\text { Specimen Dimension } \\
\left(\mathbf{m m}^{3}\right)\end{array}$ & $\begin{array}{c}\text { Clamping Length } \\
(\mathbf{m m})\end{array}$ \\
\hline $\begin{array}{l}\text { Transverse tension } \\
\text { Transverse compression }\end{array}$ & 6 & DIN EN ISO 527-5 & $250 \times 25 \times 2$ & 50 \\
\hline & 10 & DIN EN ISO 14126 & $140 \times 10 \times 2$ & 65 \\
12-shear & \multirow{3}{*}{ Puck and Schürmann [34] } & $D_{i}=70 \mathrm{~mm}$ \\
& & & $L=73 \mathrm{~mm}$ & 50 \\
\end{tabular}

The relations of technical stresses to technical strains obtained from the transverse tensile and compression tests of the UD composite are shown in Figure 4. The according shear test results are shown in Figure 5. As can be seen from the graphs, the UD composite behaves more nonlinearly under transverse compression and 12-shear than under transverse tension loading. This indicates that it is important to consider the nonlinearity and the tension/compression-asymmetry of the pure resin material behavior for the prediction of the UD composite stress response under transverse loading.

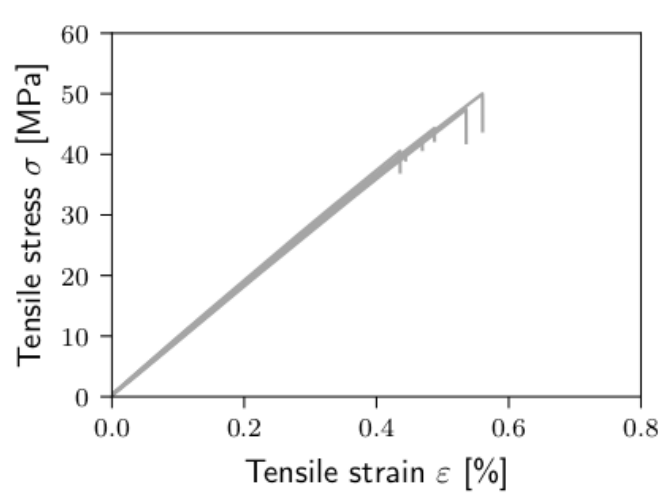

(a)

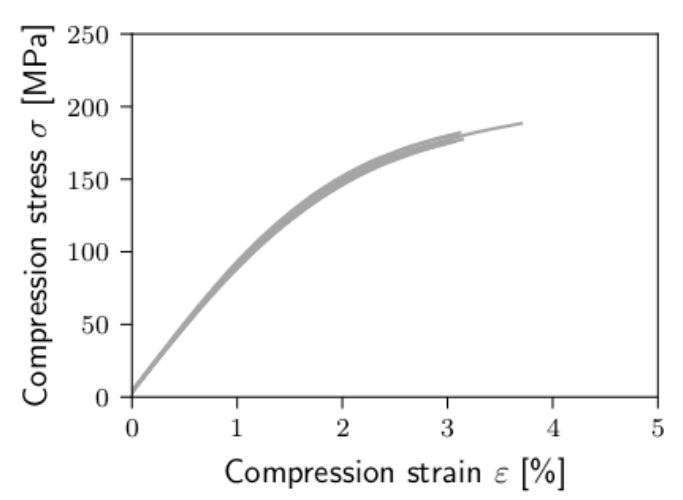

(b)

Figure 4. Experimentally observed behavior of UD composite subjected to transverse strain loading in 2-direction: (a) transverse tension and (b) transverse compression. 


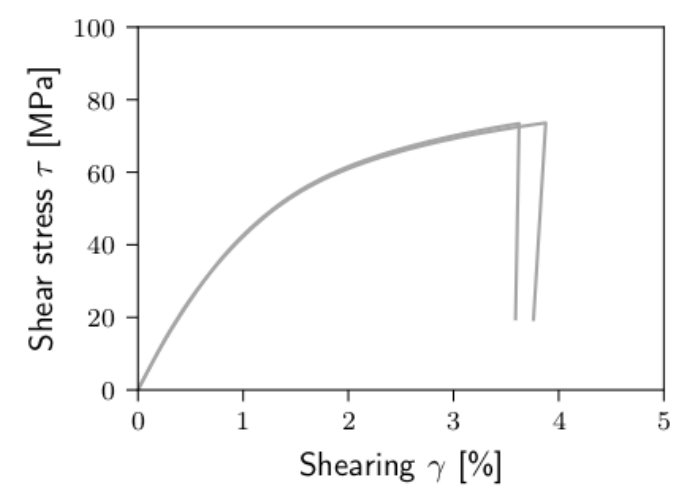

Figure 5. Experimentally observed behavior of UD composite subjected to 12-shear strain; experimental data used with kind permission of the IABG.

\section{Micromechanical Modeling Using a Representative Volume Element}

For the FEM simulations, the microstructure of the UD composite is in this work simplified to be a regular, hexagonal array despite the actually randomized fiber arrangement. This approach is used by many researchers $[9,12,25,26,28]$. In contrast to randomized fiber arrangements, the RVE size of regular arrangements can be chosen significantly smaller and there is no need for statistically validating the simulation results as there is no statistic variation of the geometry. Figure 6 shows a regular hexagonal fiber arrangement and the resulting RVE model with a fiber volume fraction (FVF) of $62 \%$. Since periodic boundary conditions are applied, using the implementation by [15] (a short description is given in Appendix A), the model is not reduced to the smallest representative unit (red border). Instead, the hexagonal RVE consists of four unit cells.

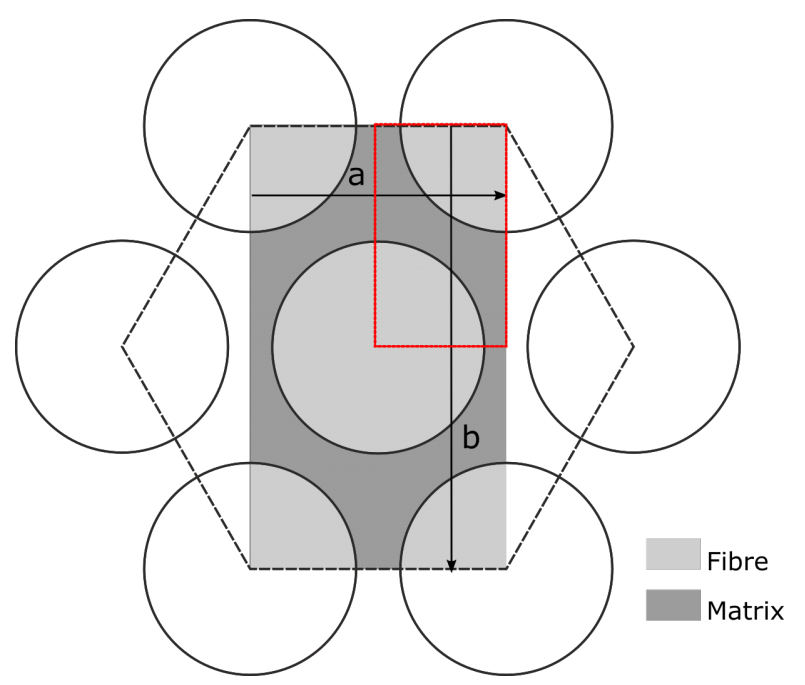

Figure 6. RVE of a hexagonal fiber arrangement with $62 \%$ fiber volume fraction; red border shows smallest representative unit.

With the fiber diameter of the IM7-fiber $\left(d_{f}=5.2 \mu \mathrm{m}\right)$ and the fiber volume fraction $(62 \%)$ the outer dimensions $a$ and $b$ of the hexagonal RVE are obtained from

$$
b=\frac{d}{2} \cdot \sqrt{\frac{2 \pi}{\tan 30^{\circ} \cdot F V F}} \quad a=b \cdot \tan 30^{\circ}
$$

For meshing, 8-node brick elements with reduced integration are used. 180 elements around the fiber circumference has proven to be a suitable compromise between accuracy and computation time. This results in an element size of $0.9 \mu \mathrm{m}$, which is also given for the meshing of the matrix. 
The dimension $c$ of the hexagonal RVE in the fiber direction is equal to the thickness of three elements, which is $2.7 \mu \mathrm{m}$.

Due to the symmetrical morphology of the hexagonal fiber arrangement, a symmetrical load-such as transverse tension or transverse compression-produces a symmetrical stress distribution in the matrix. This leads to damage initiation at all geometrically critical positions and to a symmetric crack pattern. To trigger the development of experimentally observed pronounced cracks [35] also in the simulation, model imperfections need to be incorporated. Please note that this would not be necessary for irregular fiber arrangements. For the regular hexagonal RVE this imperfection is introduced by a variation of the matrix strength in the range of $\pm 2 \%$ over all material points.

\section{Constitutive Models of the Phases}

The composite model consists of two phases: IM7 carbon fibers and an epoxy resin. The interface is modeled to be an ideal connection between fiber and matrix. The material models for fiber and matrix are implemented as user-defined material (UMAT) for the FEM-software ABAQUS ${ }^{\odot}$.

\subsection{Carbon fibers}

The carbon fiber behavior is considered transversely isotropic and linear-elastic until failure. The material-1-direction is defined longitudinal to the fiber, the material-2- and 3-orientations are defined transversely, as is shown in Figure 7. For failure detection, a maximum stress criterion [36] is suitable. This checks, whether the longitudinal fiber stress $\sigma_{11}$ exceeds the tensile strength $X_{f, 11}^{t}$ (if $\sigma_{11}>0$ ) or the compressive strength $X_{f, 11}^{c}$ (if $\sigma_{11} \leq 0$ ), respectively. As soon as fiber failure is initiated, the Young's moduli of the fiber are reduced to $0.1 \%$ of the corresponding initial value. The material parameters are taken from [32] and are given in Table 2.

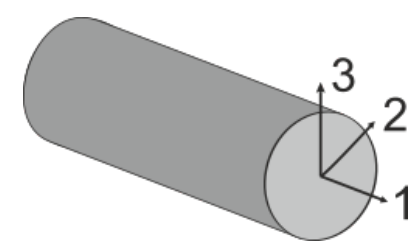

Figure 7. Coordinate system for fiber material orientations.

Table 2. Mechanical material parameters of IM7-fiber from [32].

\begin{tabular}{lccccc}
\hline Material Direction $\boldsymbol{i j}$ & Unit & $\mathbf{1 1}$ & $\mathbf{2 2 , 3 3}$ & $\mathbf{1 2 , 1 3}$ & $\mathbf{2 3}$ \\
\hline Modulus $E_{i i}, G_{i j}$ & $\mathrm{MPa}$ & 276,000 & 19,000 & 27,000 & 7000 \\
Tensile strength $X_{f, 11}^{t}$ & $\mathrm{MPa}$ & 5180 & & & \\
Compressive strength $X_{f, 11}^{c}$ & $\mathrm{MPa}$ & 3200 & & & \\
Poisson's ratio $v_{i j}$ & - & & & 0.2 & 0.357 \\
\hline
\end{tabular}

\subsection{Epoxy Resin}

The stress-strain-curves obtained by tension, compression and shear tests (Section 2) depicted in Figures 2 and 3 show a distinctly nonlinear behavior which is different for the three load cases. Kästner [28] describes the nonlinear-elastic part of the polymeric material behavior by:

$$
\sigma=\underbrace{\frac{E^{0}}{1+\delta \cdot|\varepsilon|}}_{E} \cdot \varepsilon
$$

In Equation (2), $E^{0}$ is the initial Young's modulus and $\delta$ controls the degree of nonlinearity. Another example for modeling a similar material nonlinearity by a pure elastic model is presented by 
Merodio and Haughton [37]. They use the neo-Hookean material model extended by an exponential softening term to capture a strain softening behavior.

The results of the stress calculation using model Equation (2) and the fitting parameters given in Table 3 are compared to the experimental data from the pure resin tests in Figures 8 and 9. A good approximation is obtained for the tensile stress-strain-curve and for the lower strain regime of the stress-strain behavior under compression and shear loading. The model cannot capture the stress plateau under shear and the stress decrease after the local minimum of $105 \mathrm{MPa}$ under compression. The influence of these deviations on the micromechanical simulation results for the composite is discussed in Section 5. While the initial Young's modulus $E^{0}$ is equal for tension and compression, the nonlinearity parameters $\delta^{n, t}$ and $\delta^{n, c}$ are different, ref. Table 3. Considering the different nonlinearity parameters in the elastic model, the tension/compression-asymmetry can be captured. To be able to differentiate between tension and compression for the normal strain components during the stress calculation, the sign of each strain component is checked. The corresponding Young's moduli $E_{i i}$ are calculated using Equation (2) with $E^{0}$ and $\delta=\delta^{n, t}$ for a tensile strain $\left(\varepsilon_{i i} \geq 0\right)$ and $\delta=\delta^{n, c}$ for a compressive strain $\left(\varepsilon_{i i}<0\right)$. This component-wise calculation, in the simplest way, leads to an orthotropic material model. Assuming isotropy in the lower strain range, the initial shear modulus $G^{0}$ is obtained by

$$
G^{0}=\frac{E_{0}}{2(1+v)}
$$

using the Poisson's ratio of the epoxy $v=0.395$. The nonlinearity parameter $\delta^{s}$ for shear is fitted to the experimental shear data. Thus, the strain-dependent Young's and shear moduli are obtained by:

$$
E_{i i}=\frac{E^{0}}{1+\delta^{n} \cdot\left|\varepsilon_{i i}\right|} \quad G_{i j}=\frac{G^{0}}{1+\delta^{s} \cdot|\gamma|}
$$

For orthotropic material behavior, three of the six Poisson's ratios $v_{i j}$ are independent and complete the set of nine independent material parameters. The other Poisson's ratios are determined by

$$
v_{i j} \cdot E_{j j}=v_{j i} \cdot E_{i i}
$$

Since the Young's moduli are dependent on the current strain, the Poisson's ratios vary along the path. From the experiments, however, only the constant Poisson's ratio in the range of small strains is known. If the independent Poisson's ratios-e.g., $v_{i j}$-are set to this constant value of $v_{0}=0.395$, only the dependent Poisson's ratios $v_{j i}$ would vary along the path, which is not plausible. Therefore, the mean value of $v_{i j}$ and $v_{j i}$ is assumed to be constant and is set equal to the measured value of $v_{0}=0.395$ :

$$
\frac{v_{i j}+v_{j i}}{2}=v_{0}
$$

Thus, all Poisson's ratios vary along the path, but the mean value of the corresponding ratios remains constant. The Poisson's ratios are obtained by the measured value $v_{0}$ and the current Young's moduli:

$$
v_{j i}=\frac{2 v_{0}}{\frac{E_{i i}}{E_{j j}}+1} \quad v_{i j}=2 v_{0}-v_{j i}
$$

Failure initiation in the matrix is indicated by the failure criterion by Stassi-D'Alia [38] and Raghava et al. [39]. For calibration, tensile $X_{m}^{t}$ and compressive $X_{m}^{c}$ strength are needed. As the proposed nonlinear model cannot capture the stress-strain-curves of the material at higher strains perfectly, the average maximum stress values of the experimental curves $\left(X_{m}^{t}=82 \mathrm{MPa}, X_{m}^{c}=262 \mathrm{MPa}\right)$ cannot be used as strength values for the failure criterion. In tension, this would underestimate the failure strain. The compressive stress, calculated by Equation (2) using the compressive fitting parameters, asymptotically approximates a value of $225 \mathrm{MPa}$, which is quite lower than 
the experimental maximum compressive stress value of $X_{m}^{c}=262 \mathrm{MPa}$. Thus, the modeled stresses could never reach this value. That is why "virtual" strengths are defined to compensate for the poor fit quality in the higher strain regimes, as visualized in Figure 8. The experimentally determined average failure strains for uniaxial tension $\left(\varepsilon_{f}^{t}=5.71 \%\right)$ and unaxial compression $\left(\varepsilon_{f}^{c}=37.42 \%\right)$ are inserted into Equation (2) and the obtained stress values give the "virtual" strength values in tension $X_{e q}^{t}$ and compression $X_{e q}^{c}$, respectively. The values are given in Table 3. After failure is indicated, all Young's and shear moduli are set to $d=0.1 \%$ of their initial values:

$$
E_{i i}=d \cdot E^{0} \quad G_{i j}=d \cdot G^{0}
$$

The nonlinearity parameters $\delta$ are set to zero. In quasi-static simulations, especially for the transverse compression and the shear load case of the FRP, the convergence rate of the numerical solver can deteriorate as soon as first damage occurs. That is why the viscous regularization scheme of Lapczyk and Hurtado [40] is adopted to the sudden degradation approach. The degradation parameter $d$ in Equation (8) is replaced by a regularized degradation parameter $d_{v}$. This is calculated by Equation (9).

$$
\left(1-d_{v}\right)=\frac{\Delta t}{\eta_{v}+\Delta t}\left(1-d^{k}\right)+\frac{\eta_{v}}{\eta_{v}+\Delta t}\left(1-d_{v}^{k-1}\right)
$$

It depends on the viscosity parameter $\eta_{v}$, on the current time increment $\Delta t$, on the regularized degradation parameter of the past increment $k-1$ and on the non-regularized degradation factor of the current increment $d^{k}$. Applied to the sudden degradation approach, $d^{k}$ is the defined degradation factor of $d^{k}=d=0.1 \%$.

Table 3. Material and model parameters of epoxy resin.

\begin{tabular}{llccc}
\hline Load Case & Unit & Tension & Compression & Shear \\
\hline Initial modulus $E^{0}, G^{0}$ & $\mathrm{MPa}$ & 3748 & 3748 & 1343 \\
Parameter of nonlinearity $\delta$ & - & 24.617 & 16.625 & 12.637 \\
Virtual strength $X_{\text {eq }}$ & $\mathrm{MPa}$ & 89 & 194 & - \\
\hline
\end{tabular}

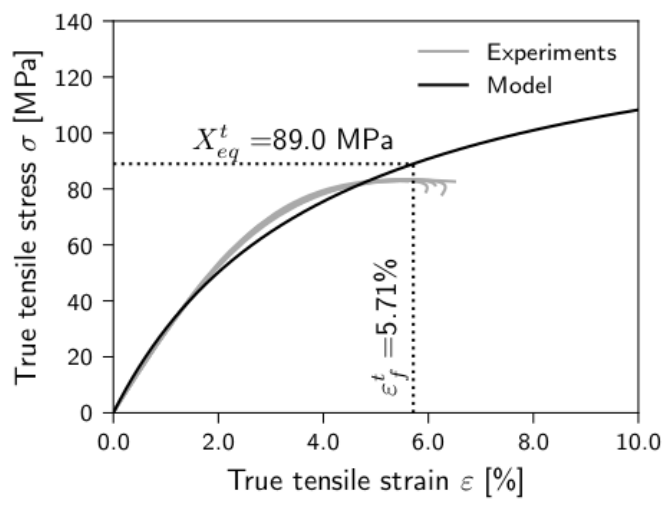

(a)

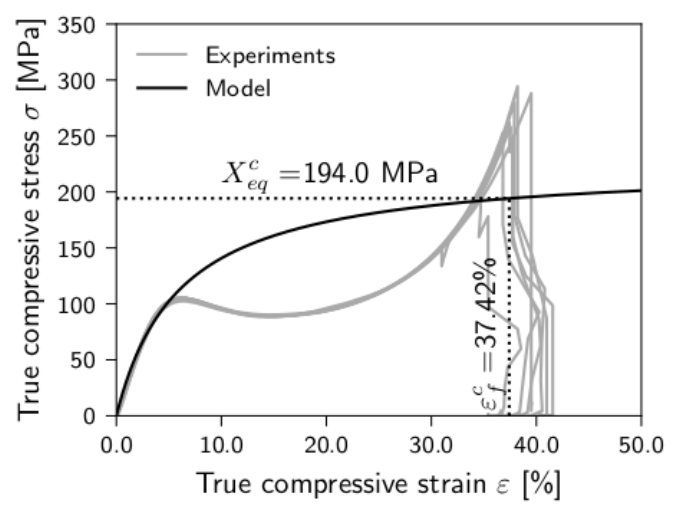

(b)

Figure 8. Experimentally observed and modeled (Equation (2)) stress-strain behavior of the epoxy resin under uniaxial normal loading: (a) tension and (b) compression. 


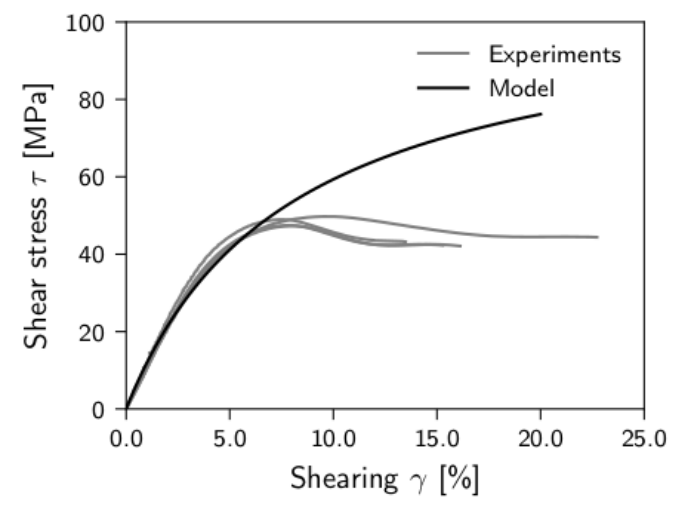

Figure 9. Experimentally observed and modeled (Equation (2)) stress-strain behavior of the epoxy resin under shear loading.

\section{Micromechanical Stiffness and Strength Prediction}

The ability of the proposed micromechanical model to predict the effective composite behavior is verified for the load cases shown in Figure 10. Under transverse tension, transverse compression and 12-shear the matrix behavior has a significant influence on the effective composite behavior. Therefore, they are suitable test cases for the assessment of the matrix modeling approach. Additionally, experimental data are available for these load cases (Section 2) and provide a validation data base.

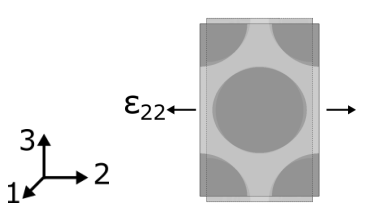

(a)

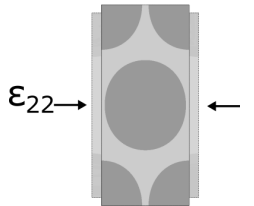

(b)

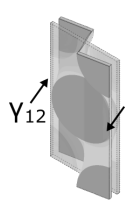

(c)

Figure 10. Matrix-dominated load cases for the validation of micromechanical modeling: (a) transverse tension, (b) transverse compression and (c) 12-shear.

The simulations are performed quasi-static (without considering inertial forces) and using an implicit solver. The load is applied to the RVE as a global strain condition. Details about applying this global strain condition and about the extraction of the effective composite stress from the micromechanical simulations results can be found in Appendix A.

For the simulation of the transverse tension load case, convergence is achieved without a viscous regularization. For transverse compression and 12-shear, the viscosity parameter must be set to $\eta_{v}=0.1$ or $\eta_{v}=0.01$, respectively.

The micromechanical prediction of the effective transverse behavior of the composite is graphically compared to the experiments in Figure 11. The figure shows the obtained effective (global) composite stress composite versus the effective (global) composite strain. The same is given for 12-shear in Figure 12. The predicted and measured composite properties and the relative errors of the predictions are given in Table 4.

Quantitative assessment of the prediction quality of micromechanical simulations is often limited to the error calculation for the composite properties as initial moduli, strengths and failure strains.

To evaluate the prediction quality for the overall nonlinear stress-strain behavior of the composite, at 100 discrete strain points within the strain range of the stress-strain-curves, the relative error of the corresponding simulated stress to the average experimental stress is calculated. This point-wise evaluation is done for the pre-damage part of the stress-strain-curve and is limited to the strain range which is covered by both simulation and experiment. In Table 4 the mean and maximum value of these point-wise evaluated relative errors is given for each load case by their amounts. 


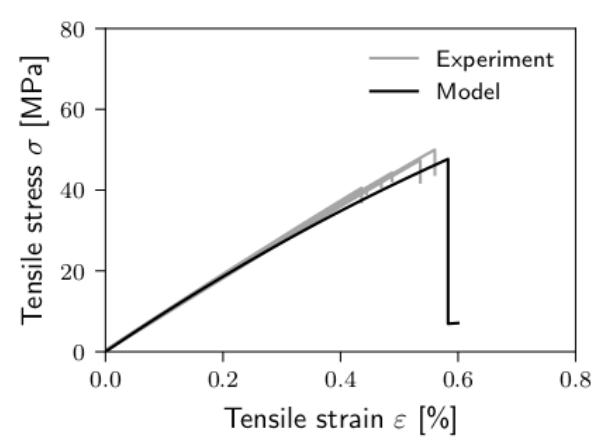

(a)

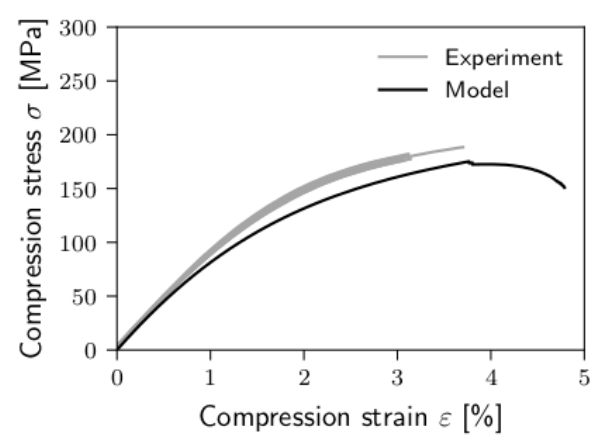

(b)

Figure 11. Micromechanical prediction of the composite behavior subjected to transverse strain loading in 2-direction: (a) transverse tension and (b) transverse compression.

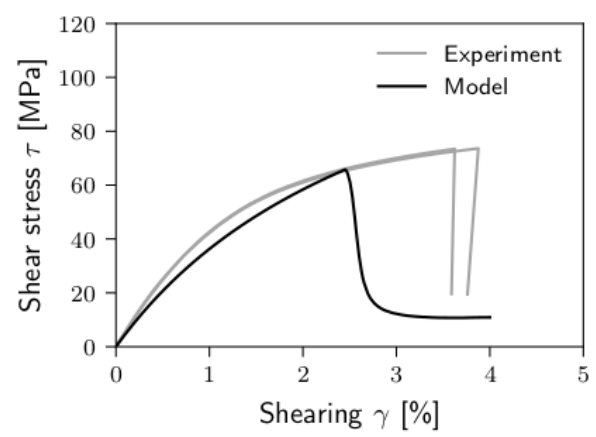

Figure 12. Micromechanical prediction of the composite behavior subjected to 12-shear strain; experimental data used with kind permission of the IABG.

The initial moduli are captured well for all load cases. The maximum relative error of $12 \%$ is obtained for the 12-shear modulus. The nonlinearity of the stress-strain behavior is systematically overestimated for transverse tension and transverse compression.

Table 4. Comparison of the micromechanical prediction of composite properties with the experimentally determined parameters.

\begin{tabular}{lrrrr}
\hline Material Property Composite & Unit & Simulation & Experiment & Relative Error \\
\hline Transverse tension modulus & $\mathrm{MPa}$ & 9327 & $9163 \pm 122$ & $2 \%$ \\
Transverse tension strength & $\mathrm{MPa}$ & 47.7 & $44.1 \pm 4$ & $8 \%$ \\
Transverse tension failure strain & & $0.58 \%$ & $0.49 \%$ & $19 \%$ \\
Stress-strain behavior, mean/max error & & & $3 \% / 8 \%$ \\
\hline Transverse compression modulus & $\mathrm{MPa}$ & 9702 & $9477 \pm 425$ & $2 \%$ \\
Transverse compression strength & $\mathrm{MPa}$ & 175 & $179 \pm 7$ & $-2 \%$ \\
Transverse compression failure strain & & $3.77 \%$ & $3.12 \%$ & $21 \%$ \\
Stress-strain behavior, mean/max error & & & & $10 \% / 12 \%$ \\
\hline 12-shear modulus & $\mathrm{MPa}$ & 4539 & $5140 \pm 981$ & $-12 \%$ \\
12-shear strength & $\mathrm{MPa}$ & 66 & $72 \pm 11$ & $-8 \%$ \\
Failure shearing & & $2.45 \% 1$ & $3.75 \%$ & $-35 \%$ \\
Stress-strain behavior, mean/max error & & & $11 \% / 18 \%$ \\
\hline
\end{tabular}

${ }^{1}$ Experimental data used with kind permission of IABG.

The best fit of the experimentally and the numerically obtained stress-strain-curves is obtained for transverse tension. The relative mean and maximum deviation of the numerical and experimental curves are quite low (3\% and $8 \%$, resp.). The deviation is higher for transverse compression behavior 
and for the 12-shear load case, ref. Table 4, but the model prediction is still within an acceptable range (maximum error of $18 \%$ ).

For the pure resin, the model significantly deviates from the experiments for strains higher than $6 \%$ for compression and for shearing higher than $7 \%$ for shear load, respectively (Figures $8 \mathrm{~b}$ and 9 ). These deviations do not affect the quality of the micromechanical simulations of the composite to a relevant extent. Figure 13a shows the local matrix strain distribution for a transverse compression load case in a composite one load increment before failure initiates. In the areas highlighted in black, the local strain component in the direction of the external load exceeds $6 \%$. This is the case for about half of the matrix area. Due to the error of the pure resin model, a significant overestimation of the stresses in the black highlighted areas could be suspected. Due to the extent of these areas, this should lead to a significant overestimation of the effective composite stresses. This is, however, not observed (Figure 11b). To find an explanation, Figure 13b shows the local stress distributions in terms of the ratio of the first stress invariant $I_{1}$ to the von Mises equivalent stress $\sigma_{V M}$. The first invariant gives the dilatoric part or hydrostatic pressure $p\left(I_{1}=3 \cdot p\right)$ of a stress state. The von Mises equivalent stress is a measure for the deviatoric part. For a uniaxially stressed homogeneous material, the first invariant and the von Mises equivalent stress are equal. For multiaxial stress states, as found in the matrix in a composite under transverse compression load, the hydrostatic pressure is much higher than the deviatoric part. Only in a few areas of the matrix (highlighted in black in Figure 13b) the equivalent stress according to von Mises is equal to or greater than the first invariant. The plastic behavior of the epoxy resin, which is often assumed to alone cause the nonlinear matrix material behavior, depends on the hydrostatic pressure component of the stress state [21,29]. Higher hydrostatic pressure raises the yield point of the material. So, although plastic flow occurs in the epoxy resin under uniaxial compressive loading from a certain strain, in the composite, this is delayed by the higher hydrostatic stress component. Thus, local plasticity of the matrix seems to be less relevant for the global composite stress-strain behavior.

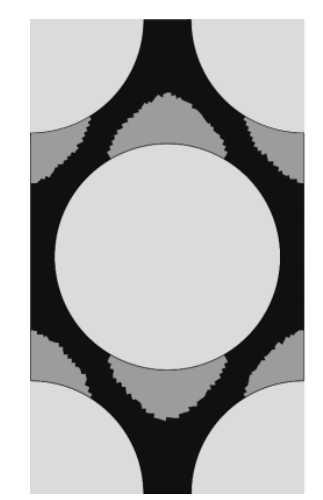

(a)

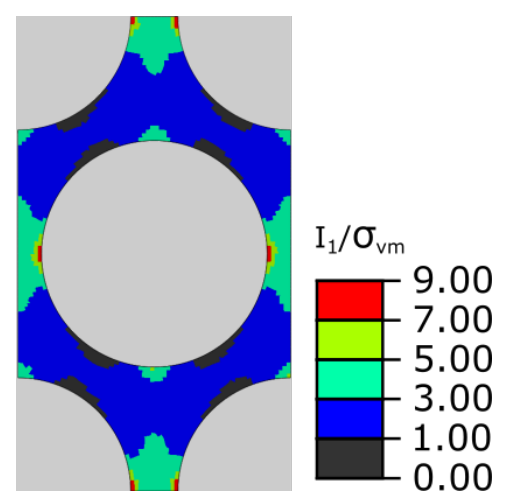

(b)

Figure 13. Local stress and strain distribution in the matrix of a composite loaded in transverse compression, one increment before local failure initiates: (a) amount of local matrix strain in the direction of the external load and (b) ratio of the first invariant $I_{1}$ and the von Mises equivalent stress $\sigma_{V M}$.

Altogether, the micromechanical simulation with nonlinear-elastic and orthotropically modeled matrix satisfactorily predicts the stiffness behavior of the UD composite. The consideration of the nonlinearity and the tension/compression-asymmetry seems to have the highest impact on the prediction quality of the composite stiffness. Whether these phenomena are attributed to and modeled with nonlinear-elastic or elasto-plastic effects, is of secondary importance. This also seems to be true for the global composite strengths: The maximum relative error by amount of the predicted strengths is $8 \%$, Table 4 . 
The failure strain — defined as strain value corresponding to the stress maximum —is slightly overestimated for the transverse tension and compression load case (Table 4). For shear, the failure strain is underestimated ( $2.45 \%$ instead of $3.75 \%)$. In addition to the differences between the calculated and the measured strengths, this is also due to the over- or underestimation of the material nonlinearity. Compared to the transverse tension load case, this deviation is somewhat higher for transverse compression and 12-shear.

The simulated matrix damage, shown in Figure 14, initiates in matrix elements adjacent to the fibers, which is in accordance with experimental observations.

As the interface is modeled to be a perfect connection of fiber and matrix, damage occurs only within the matrix phase. Strictly speaking, there is no interface failure in the model. Nevertheless, the failure of the matrix elements being directly connected to the fiber elements is referenced to "interface damage" or "debonding", in the following. The contour plots in Figure 15 explain why the damage initiates at the fiber-matrix-interface. The plots show the matrix stress distribution within the transversally loaded UD composite one load increment before damage initiates. The matrix failure behavior is pressure-dependent: The higher the amount of a negative dilatoric stress (hydrostatic pressure $p<0$ ), the higher the bearable amount of the deviatoric stress part, represented by $\sigma_{V M}$. Thus, for stress states with positive dilatoric stresses $(p \geq 0)$, these dominate the matrix failure. For stress states with negative dilatoric stresses, the failure becomes deviatorically dominated. Figure 15a shows the dilatoric matrix stress distribution in the composite under transverse tensile load. The dilatoric stresses are positive over the whole RVE. The opposite is true for the other load cases: Under transverse compression, mainly negative hydrostatic matrix stresses are present and under shear the hydrostatic stress proportion is negligibly small. Therefore, Figure $15 b, c$ show the distribution of the von Mises equivalent stress $\sigma_{V M}$ as this dominates the matrix failure for these load cases. Due to the property mismatch of fiber and matrix, stress concentrations (red areas) arise in these regions for all load cases. Within these red areas, the damage initiates in the matrix elements which are directly connected to the fibers. Due to the introduced local scatter of the matrix strength, the damage is not initiated in all (red) areas with stress concentration but only in the elements with a critical combination of high stresses and small strength.

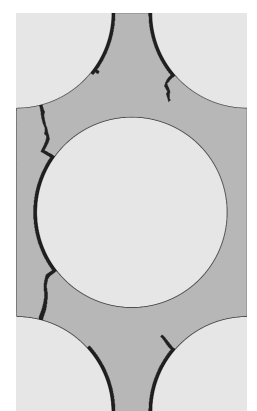

(a)

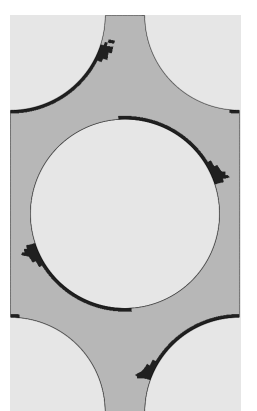

(b)

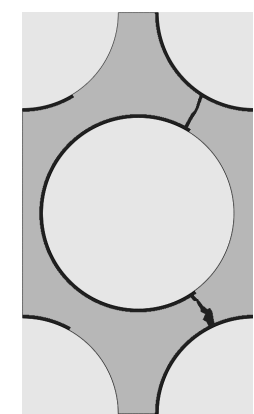

(c)

Figure 14. Simulated crack patterns at (a) transverse tension, (b) transverse compression and (c) 12-shear loading; black areas indicate matrix damage. 


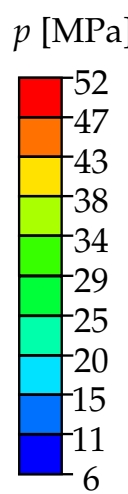

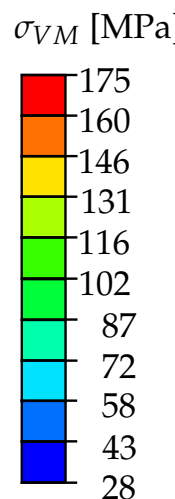

(a)

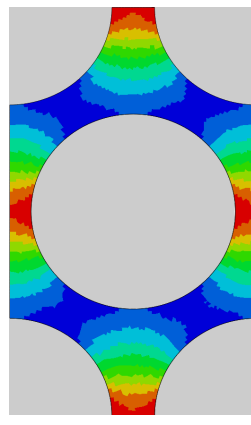

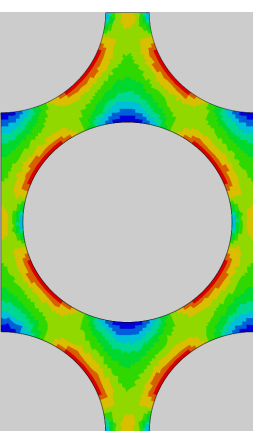

(b)

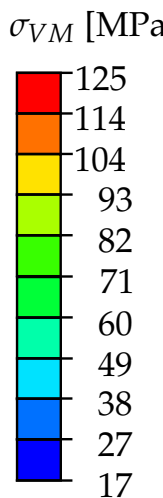

17

(c)

Figure 15. Local matrix stress distribution one load increment before damage initiates under (a) transverse tension (b) transverse compression and (c) 12-shear loading.

This "debonding" grows until the damage changes direction and connects the interface damages through the matrix. Under transverse tension, the crack forms parallel to the load direction, as experimentally observed $[10,35]$. Under transverse compression the simulation shows a matrix crack that grows oblique to the loading direction. This is also experimentally observed by [41] as well as by [10]. Under 12-shear loading, the fiber-matrix-debondings grow very far around the fiber before they connect through the matrix region. In the calculations by [9-11], the debonding is less pronounced before it grows into the matrix region to connect with neighboring debonds.

\section{Prediction Quality Compared to Literature Models}

After the error of the model prediction has been assessed, the prediction quality is compared to similar models found in the literature. For this comparison, published micromechanical simulation results based on different material modeling approaches are selected that have also been compared to experimental data. As the present paper is not a review, this model selection does not claim completeness. Most of the selected models use an elasto-plastic [7,9-11] or visco-plastic $[12,13,27,28]$ modeling approach for the pure resin material. Two micromechanical simulations based on a linear-elastic and isotropic material model of the matrix are represented [14,23]. The cited micromechanical approaches also differ concerning the modeled fiber arrangement and the interface modeling. Table 5 provides the key aspects of the micromechanical modeling approach for each selected model.

Table 5. Key aspects of the micromechanical literature models selected for comparison.

\begin{tabular}{|c|c|c|c|c|}
\hline Model & Composite & Fiber Arrangement & Material Modeling & Interface Modeling \\
\hline Bai et al. [27] & C/Epoxy & randomized & visco-plastic & perfectly bonded \\
\hline Ernst et al. $[9,25]$ & G/Epoxy & square & elasto-plastic & perfectly bonded \\
\hline Govaert et al. [12] & G/Epoxy & hexagonal & elasto-plastic & perfectly bonded \\
\hline Kästner [28] & $\mathrm{G} / \mathrm{PP}$ & hexagonal & visco-plastic & perfectly bonded \\
\hline Okabe et al. [13] & C/Epoxy & randomized & elasto-plastic & perfectly bonded \\
\hline Ren et al. [14] & C/Epoxy & hexagonal & linear-elastic & perfectly bonded \\
\hline Riaño et al. [22] & G/Epoxy & randomized & linear-elastic & continuum mechanics \\
\hline Sun et al. [10] & C/Epoxy & randomized & elasto-plastic & combined ${ }^{1}$ \\
\hline Tan et al. [11] & C/Epoxy & randomized & elasto-plastic & cohesive zone \\
\hline Vaughan and McCarthy [7] & C/Epoxy & randomized & elasto-plastic & cohesive zone \\
\hline presented model & C/Epoxy & hexagonal & nonlinear-elastic & perfectly bonded \\
\hline
\end{tabular}

For more details, the reader is referred to the individual publications. The key aspects of the herein presented model are summarized in the table, as well. 
For each selected model, the given experimental and simulation results are extracted from the publications and the relative error of the simulation with respect to the experiment is computed in the same manner as has been done for the herein the presented results in Section 5. It should be noted that the publications treat different material systems (fiber and matrix type are also given in Table 5) and, thus, each simulation is compared to a different experimental data base. The probably different scattering of these data bases also effects the estimated relative errors of the model predictions. Nevertheless, it is assumed that the calculated relative errors provide a good estimation of the accuracy of the individual models.

In the following, the simulation errors of the selected literature models are compared to each other (bar plots in Figures 16-18). Additionally, the simulation errors obtained within the herein presented study are also displayed in the figure (black dashed line in Figures 16-18). As the prediction of initial modulus is usually reasonably accurate, only the relative error for the predicted stress-strain behavior and for the strength prediction are compared. Not every selected publication provides simulation results for all composite properties or delivers the complete stress-strain-curve for the considered load cases. Because of that, not every model is represented in each diagram. Most micromechanical simulation results are presented for the transverse tension behavior, Figure 16. The bar plots give the relative prediction errors for each individual literature model with respect to the corresponding experiments. Additionally, the black dashed line shows the relative prediction error of the herein present model. Regardless of whether the proposed model over- or underestimates the experimental value, the error is plotted on both the positive and the negative y-axis.

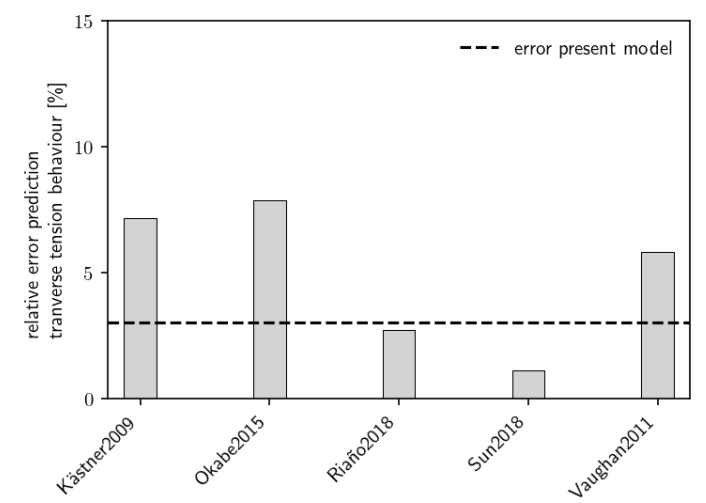

(a)

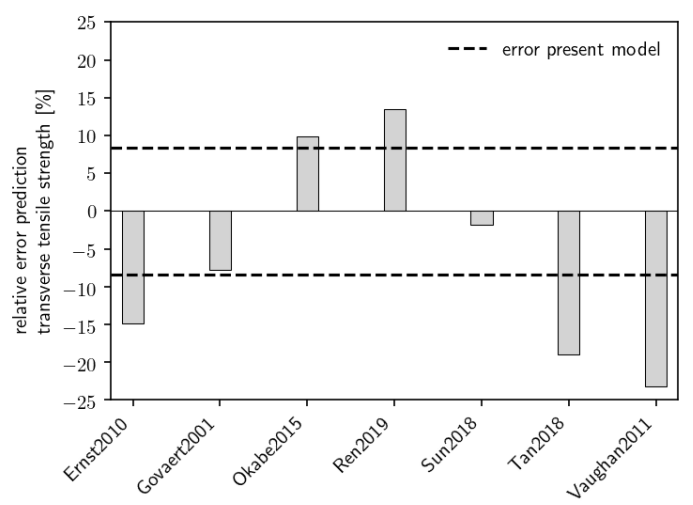

(b)

Figure 16. Relative error for prediction of (a) transverse tension stress-strain behavior and (b) transverse tensile strength with models from the literature $[7,10-14,23,25,28]$ and with the herein the presented model.

For the prediction of the transverse tension behavior, two ( Sun et al. [10], Riaño et al. [23]) out of five models give a more accurate prediction regarding the relative error than the herein presented model. For the transverse tensile strength, two ( Sun et al. [10], Govaert et al. [12]) out of seven models yield to a better prediction. Thereby, only Sun et al. [10] provides a comparison for both the elastic behavior and the strength. 


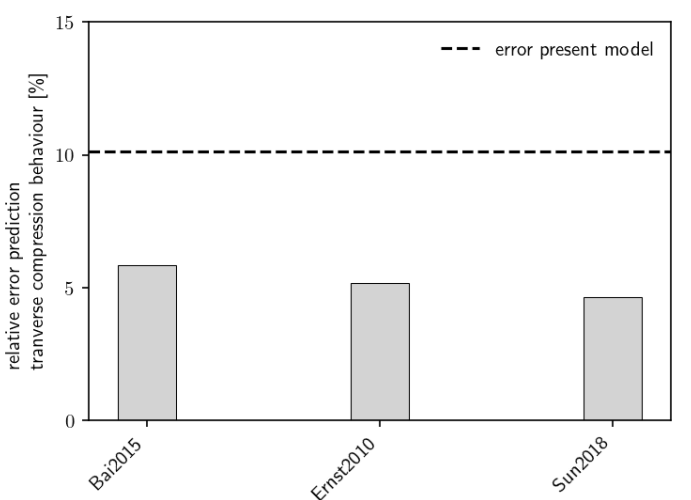

(a)

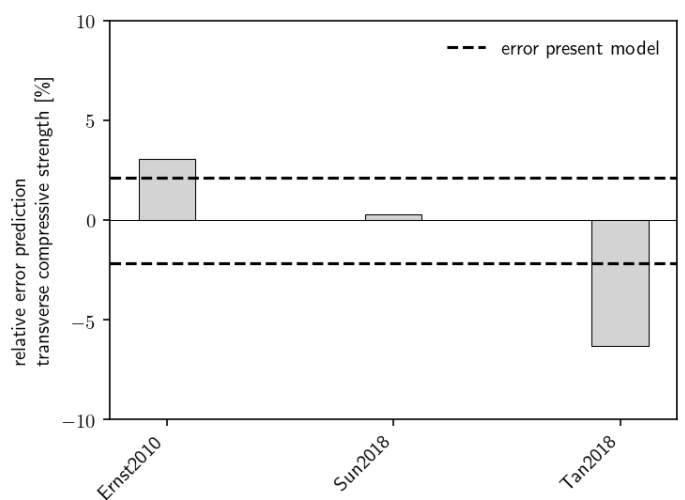

(b)

Figure 17. Relative error for prediction of (a) transverse compression stress-strain behavior and (b) transverse compressive strength with models from the literature $[10,11,25,27]$ and with the herein presented model.

For the transverse compression behavior, the relative error of $10 \%$ is higher than the relative errors of all selected literature models, Figure $17 \mathrm{~b}$. The transverse compressive strength prediction of the herein presented model is, one the other hand, more accurate than two out of three selected models, Figure 17a.

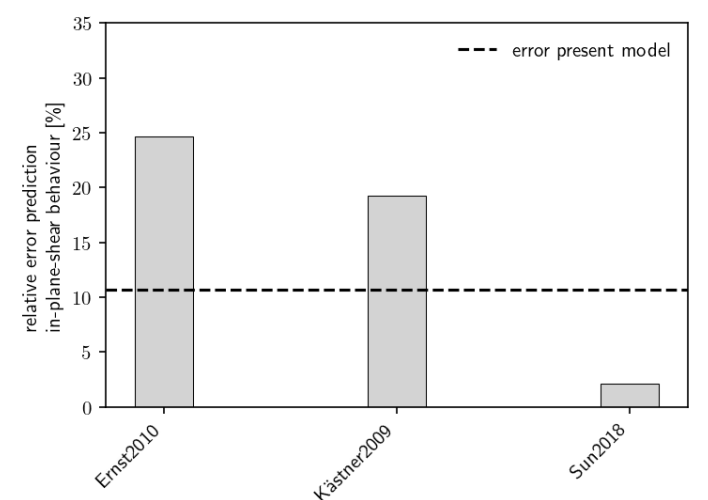

(a)

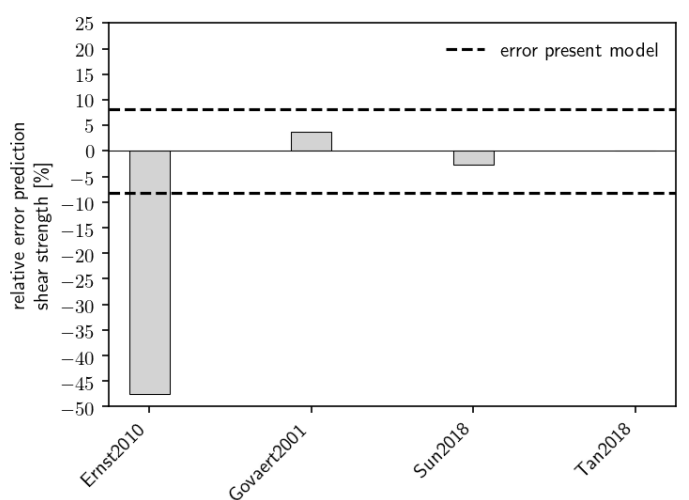

(b)

Figure 18. Relative error for prediction of (a) the 12-shear stress-strain behavior and (b) 12-shear strength with models from the literature $[10-12,25,28]$ and with the herein presented model; error of shear strength prediction by Tan et al. [11] is $0.1 \%$.

For the prediction of the 12-shear behavior and strength the models by Sun et al. [10] and by Tan et al. [11] provides particularly good results, which are quite better than the herein presented predictions, Figure 18. The relative errors of the models by Ernst et al. [9] and Kästner [28] are quite high $(>15 \%)$ and higher than the errors of the herein presented model. In general, there is a significant greater difference in the model prediction quality of the literature models for the 12-shear load case than for the other matrix-dominated load cases. Overall, the herein presented model gives a good accuracy compared to the literature models. However, the comparison between simulation and experiment must not be regarded as validation for all models presented in the literature. Sun et al. [10] and Okabe et al. [13], whose model leads to very good prediction results, use some experimental composite data for a reverse calibration of the matrix failure criterion or the fiber-matrix-interface parameters, respectively. Vaughan and McCarthy [7,8] do not use experimental composite data directly for calibration, 
but they compare the simulation results with different interface parameters. Figure 16 shows the error for the simulation result closest to the experiments. The reverse model calibration reduces the validation database. Depending on the goal of the micromechanics simulation, e.g., if parametric studies in terms of the model parameters are conducted, this procedure is expedient. On the other hand, a prediction of the composite behavior is only possible if experiments on the composite level are not used to adjust the micromechanical model parameters. Besides the model presented here, the only for comparison selected models that make no use of a reverse calibration and give prediction results for all here considered load cases are the models by Ernst et al. [9] and Tan et al. [11].

\section{Summary and Conclusions}

The present research investigates whether an elastic material model for the epoxy matrix in micromechanical simulations sufficiently predicts the material behavior of transversely loaded FRPs. The model of the stress-strain behavior of the epoxy is strain-dependent and orthotropic in order to capture the nonlinearity and the tension/compression-asymmetry of the resin's constitutive behavior. While the representation of the neat resin's tensile behavior is sufficient, larger deviations from the experimental data occur for compression and shear at higher strains. For failure and progressive damage modeling a stress invariant-based failure criterion and a sudden material degradation together with a viscous damage regularization scheme is used. To examine the predictive capability of this model, FEM-based micromechanical simulations using a hexagonal RVE are conducted. It is focused on the matrix-dominated load cases transverse tension, transverse compression and 12-shear. To verify the model, the simulation results are compared to experimental data. Additionally, the accuracy of the presented micromechanical predictions are compared to that of other models published in the literature. The relative error of the predicted initial moduli, strengths and failure strains is evaluated. Additionally, an error measurement is proposed indicating how well the nonlinear stress-strain behavior of the composite is predicted. The relative error of the predicted composite material parameters and the predicted composite strengths is a maximum of $8 \%$. The maximum deviation of the predicted stress-strain behavior from the experiment is $18 \%$. This is a reasonably good accuracy and is within the accuracy of the literature models. This indicates the admissibility of the assumptions made. Furthermore, it seems primarily important to capture the nonlinearity and the tension/compression-asymmetry of the resin's constitutive behavior. To predict the global transverse composite behavior, it seems less important whether this is depicted by plasticity. Thus, this study gives the indication that a pure elastic model considering the named effects might be sufficient for a reasonably good micromechanical stiffness and strength prediction, if rate-dependent effects are negligible. This facilitates the numerical calculation, as elastic models do not need an iterative process within the material equations and, therefore, are numerically more efficient.

This could be further quantified with a direct comparison of different material modeling approaches calibrated and benchmarked against the same experimental data.

The importance of plastic effects on the global composite behavior could be different for composites made of other polymeric materials with a different plasticity behavior, e.g., a different pressure-sensitivity. Thus, a further research question is to verify whether the presented purely elastic modeling approach is also applicable to other resin materials. Additionally, plasticity maybe could play a more important role for the global composite behavior for other, e.g., multiaxial, load cases. Hence, it would be worth to simulate multiaxial load cases and compare the micromechanical simulation results to corresponding experiments. This could be a further evaluation of the presented modeling approach.

Funding: This research was funded by the German Research Foundation, DFG, project number 379482657 and by the Federal Ministry of Economic Affairs and Energy of Germany, project HyMod.

Conflicts of Interest: The authors declare no conflict of interest. The funders had no role in the design of the study; in the collection, analyses, or interpretation of data; in the writing of the manuscript, or in the decision to publish the results. 


\section{Abbreviations}

The following abbreviations are used in this manuscript:

C carbon fiber

CFRP carbon fiber-reinforced plastic

DIC digital image correlation

DOF degrees of freedom

FEM finite element method

FVF fiber volume fraction

FRP fiber-reinforced plastics

G glass fiber

GFRP glass fiber-reinforced plastic

IABG Industrieanlagen-Betriebsgesellschaft $\mathrm{mbH}$

PP polypropylene

RP reference point

RVE representative volume element

UMAT user-defined material

UD unidirectional

a width of representative volume element

$b \quad$ height of representative volume element

c thickness of representative volume element in fiber direction

$D_{i} \quad$ inner diameter

$D_{0} \quad$ outer diameter

d degradation factor

$d_{f} \quad$ fiber diameter

$d_{v} \quad$ viscous regularized degradation factor

$d_{v}^{k-1} \quad$ regularized degradation factor of last increment $k-1$

E Young's modulus

$E^{0} \quad$ initial Young's modulus

f nodel force

G shear modulus

$G^{0} \quad$ initial shear modulus

$\mathrm{H} \quad$ displacement gradient

$I_{1} \quad$ first invariant of stress state

$i, j \quad$ index notation of material direction

$k \quad$ current increment

L length

$P \quad$ node

$p \quad$ hydrostatic pressure

u displacement

$\tilde{u} \quad$ fluctuation

$X \quad$ strength

$X_{f, 11}^{c} \quad$ longitudinal compressive fiber strength

$X_{f, 11}^{t} \quad$ longitudinal tensile fiber strength

$X_{e q}^{c^{\prime}} \quad$ equivalent compressive strength

$X_{e q}^{t} \quad$ equivalent tensile strength

$X_{m}^{c} \quad$ matrix compressive strength

$X_{m}^{t} \quad$ matrix tensile strength

$x \quad$ position vector

$\gamma \quad$ shearing

$\delta \quad$ nonlinearity parameter

$\delta^{n} \quad$ nonlinearity parameter for normal stress

$\delta^{n, c} \quad$ nonlinearity parameter for normal compressive stress

$\delta^{n, t} \quad$ nonlinearity parameter for normal tensile stress 
$\delta^{s} \quad$ nonlinearity parameter for shear stress

$\Delta t \quad$ time increment

$\varepsilon \quad$ strain

$\varepsilon^{\text {macro }} \quad$ effective macroscopic (global) strain

$\varepsilon_{f} \quad$ failure strain

$\varepsilon_{f}^{c} \quad$ compressive failure strain

$\varepsilon_{f}^{t} \quad$ tensile failure strain

$\eta_{v}$ parameter for viscous regularization

$v \quad$ Poisson's ratio

$v_{0} \quad$ initial Poisson's ratio

$\sigma \quad$ stress

$\sigma^{\text {macro }} \quad$ effective macroscopic (global) stress

$\sigma_{V M} \quad$ von Mises equivalent stress

$\tau$ shear stress

\section{Appendix A}

The periodic boundary conditions of the RVE are applied by constraints between opposite nodes on the RVE's surface [42]. The displacements $u_{i}$ of two opposite nodes $P^{+}$and $P^{-}$with their position vectors $x_{j}^{+}$and $x_{j}^{-}$are defined by

$$
\begin{aligned}
& u_{i}^{+}=H_{i j} x_{j}^{+}+\tilde{u}_{i} \\
& u_{i}^{-}=H_{i j} x_{j}^{-}+\tilde{u}_{i}
\end{aligned}
$$

Herein, $H_{i j}$ denotes the displacement gradient on macroscale and $\tilde{u}$ is the fluctuation. Demanding periodicity in displacements, the fluctuation is equal for both facing nodes. Subtraction of Equation (A2) from (A1) gives

$$
u_{i}^{+}-u_{i}^{-}=H_{i j}\left(x_{j}^{+}+x_{j}^{-}\right)=H_{i j} \Delta x_{j}
$$

It should be mentioned that as the nodes are opposite to each other, their position vectors $x_{j}$ vary in only one coordinate. For the implementation of Equation (A3) into commercial FE software, additional degrees degrees of freedom (DOF) must be introduced representing the components of the (global) displacement gradient on macroscale. Three dummy nodes with three displacement DOF each are defined to represent the nine components of the displacement gradient, Figure A1. They are called reference points (RP).

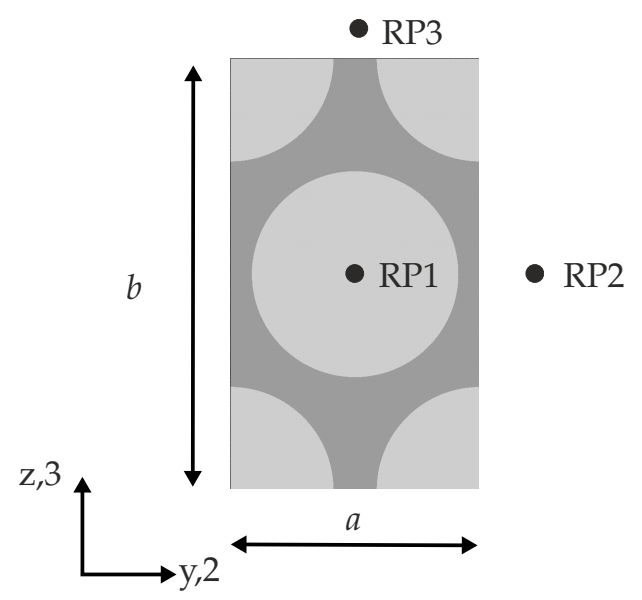

Figure A1. Additional reference points (RP) in FEM model of RVE introducing additional degrees of freedom representing the components of the displacement gradient on macroscale. 
It is comfortable to define the DOF of the first reference point to be the components $H_{i 1}$ of the displacement gradient. That means, the first DOF of the first reference point corresponds to $H_{11}$, the second corresponds to $H_{21}$ and the third corresponds to $H_{31}$. The same correlation is defined between the DOF of the second and third reference point and the displacement gradient's components $H_{i 2}$ and $H_{i 3}$. Using this definition, the general form of Equation (A3) in terms of the reference points' $\mathrm{DOF}$ is

$$
u_{i}^{+}-u_{i}^{-}=u_{i}^{R P j} \Delta x_{j}
$$

Herein, $u_{i}^{R P j}$ is DOF $i$ of reference point $j$. Implemented for each pair of opposite nodes, the constraint of Equation (A4) realizes the periodic boundary conditions of the RVE.

For deforming the RVE, displacements or forces are applied to the DOF of the reference points which than represent the macroscopic loading of the FRP. Due to the coupling by Equation (A4), the nodal forces and nodal displacements of the reference points are directly related to the effective stresses and strains integrated via the volume of the RVE. To determine the effective material behavior of the RVE, it is sufficient to evaluate the nodal forces and displacements at the reference points. To give an example, a transversally loaded RVE is considered. The macroscopic tensile strain $\varepsilon_{22}^{\text {macro }}$ is applied by a prescribed displacement of $\varepsilon_{22}^{\text {macro }}$ at the second DOF of the second reference point. The effective transverse stress $\sigma_{22}^{\text {macro }}$ resulting from the applied deformation can be calculated from the resulting nodal force $\mathrm{f}_{2}^{R P 2}$ at the second DOF of the second reference point by dividing it by the RVE's volume. Please note that obtaining stresses from forces usually only requires the relevant cross-sectional area. For a transverse loading in y-direction, this would be given by $b \cdot c$, with $b$ and $c$ denoting the RVE height and thickness, respectively, Figure A1. However, since the constraining equation contains the dimension of the RVE in the loading direction as a scaling parameter, it must be considered in the stress calculation. Thus, the reference point forces have to be divided by the volume (cross sectional area $b \cdot c$ times the width $a$ of the RVE). This is also the reason the effective normal strains can be directly derived from the displacmenets of the reference points, see Equation (A5).

The following equations summarize the correlation between the nodal displacements and forces of the reference points to the effective macroscopic strains and stresses for the example of a transversally loaded RVE:

$$
\begin{gathered}
\varepsilon_{22}^{\text {macro }}=u_{2}^{R P 2} \\
\sigma_{22}^{\text {macro }}=\frac{f_{2}^{R P 2}}{a \cdot b \cdot c}
\end{gathered}
$$

When considering shear loading, note that applying loads only on the reference point DOF connected to displacement gradient component $H_{12}$ results in a simple shear configuration while applying loads at $\mathrm{H}_{12}$ and $\mathrm{H}_{21}$ results in pure shear. The effective shear strains and stresses are then computed from Equations (A5) and (A6) using the average displacement and force components of the relevant reference point DOF (in this case $u_{1}^{R P 2}$ and $u_{2}^{R P 1}$ as well as $\mathrm{f}_{1}^{R P 2}$ and $\mathrm{f}_{2}^{R P 1}$ ).

\section{References}

1. Hobbiebrunken, T.; Hojo, M.; Adachi, T.; De Jong, C.; Fiedler, B. Evaluation of interfacial strength in CF/epoxies using FEM and in-situ experiments. Compos. Part A Appl. Sci. Manuf. 2006, 37, 2248-2256. [CrossRef]

2. Jones, F.R. A Review of Interphase Formation and Design in Fibre-Reinforced Composites. J. Adhes. Sci. Technol. 2012, 24, 171-202. [CrossRef]

3. Shioya, M.; Yasui, S.; Takaku, A. Relation between interfacial shear strength and tensile strength of carbon fiber/resin composite strands. Compos. Interf. 1999, 6, 305-323. [CrossRef] 
4. Budiman, B.A.; Juangsa, F.B.; Aziz, M.; Nurprasetio, I.P.; Zaini, I.N. Experimental verification of interfacial strength effect on the mechanical properties of carbon fiber-epoxy composite. Int. J. Adv. Sci. Eng. Inf. Technol. 2017, 7, 2226-2231. [CrossRef]

5. Asp, L.E.; Berglund, L.A.; Talreja, R. Prediction of matrix-initiated transverse failure in polymer composites. Compos. Sci. Technol. 1996, 56, 1089-1097. [CrossRef]

6. Asp, L.E.; Berglund, L.A.; Talreja, R. Effects of Fiber and Interphase on Matrix- Initiated Transverse Failure in Polymer Composites. Compos. Sci. Technol. 1996, 56, 657-665. [CrossRef]

7. Vaughan, T.J.; McCarthy, C.T. Micromechanical modelling of the transverse damage behaviour in fibre reinforced composites. Compos. Sci. Technol. 2011, 71, 388-396. [CrossRef]

8. Vaughan, T.J.; McCarthy, C.T. A micromechanical study on the effect of intra-ply properties on transverse shear fracture in fibre reinforced composites. Compos. Part A Appl. Sci. Manuf. 2011, 42, 1217-1228. [CrossRef]

9. Ernst, G.; Vogler, M.; Hühne, C.; Rolfes, R. Multiscale progressive failure analysis of textile composites. Compos. Sci. Technol. 2010, 70, 61-72. [CrossRef]

10. Sun, Q.; Meng, Z.; Zhou, G.; Lin, S.p.; Kang, H.; Keten, S. Multi-scale computational analysis of unidirectional carbon fi ber reinforced polymer composites under various loading conditions. Compos. Struct. 2018, 196, 30-43. [CrossRef]

11. Tan, W.; Naya, F.; Yang, L.; Chang, T.; Falzon, B.G.; Zhan, L.; Molina-Aldareguía, J.M.; González, C.; Llorca, J. The role of interfacial properties on the intralaminar and interlaminar damage behaviour of unidirectional composite laminates: Experimental characterization and multiscale modelling. Compos. Part B Eng. 2018, 138, 206-221. [CrossRef]

12. Govaert, L.E.; Schellens, H.J.; Thomassen, H.J.M.; Smit, R.J.M.; Terzoli, L.; Peijs, T. A micromechanical approach to time-dependent failure in off-axis loaded polymer composites. Composit. Part A Appl. Sci. Manuf. 2001, 32, 1697-1711. [CrossRef]

13. Okabe, T.; Imamura, H.; Sato, Y.; Higuchi, R.; Koyanagi, J.; Talreja, R. Experimental and numerical studies of initial cracking in CFRP cross-ply laminates. Compos. Part A Appl. Sci. Manuf. 2015, 68, 81-89. [CrossRef]

14. Ren, M.; Zhang, X.W.; Huang, C.; Wang, B.; Li, T. An integrated macro/micro-scale approach for in situ evaluation of matrix cracking in the polymer matrix of cryogenic composite tanks. Compos. Struct. 2019, 216, 201-212. [CrossRef]

15. Krause, D. A physically based micromechanical approach to model damage initiation and evolution of fiber reinforced polymers under fatigue loading conditions. Compos. Part B Eng. 2016, 87, 176-195. [CrossRef]

16. Lüders, C.; Sinapius, M.; Krause, D. Adaptive cycle jump and limits of degradation in micromechanical fatigue simulations of FRP. Int. J. Damage Mech. 2019. [CrossRef]

17. Pindera, M.J.; Khatam, H.; Drago, A.S.; Bansal, Y. Micromechanics of spatially uniform heterogeneous media: A critical review and emerging approaches. Compos. Part B Eng. 2009, 40, 349-378. [CrossRef]

18. Matthias Sanden. Mathematische Homogenisierung in der Kontinuumsmechanik. Ph.D. Thesis, Technische Universität Darmstadt, Darmstadt, Germany, 2013.

19. Ramírez-Torres, A.; Penta, R.; Rodríguez-Ramos, R.; Grillo, A.; Preziosi, L.; Merodio, J.; Guinovart-Díaz, R.; Bravo-Castillero, J. Homogenized out-of-plane shear response of three-scale fiber-reinforced composites. Comput. Vis. Sci. 2019, 20, 85-93. [CrossRef]

20. Hojo, M.; Mizuno, M.; Hobbiebrunken, T.; Adachi, T.; Tanaka, M. Effect of fiber array irregularities on microscopic interfacial normal stress states of transversely loaded UD-CFRP from viewpoint of failure initiation. Compos. Sci. Technol. 2009, 69, 1726-1734. [CrossRef]

21. Fiedler, B.; Hojo, M.; Ochiai, S.; Schulte, K.; Ando, M. Failure behavior of an epoxy matrix under different kinds of static loading. Compos. Sci. Technol. 2001, 61, 1615-1624. [CrossRef]

22. Riaño, L.; Belec, L.; Joliff, Y. Validation of a Representative Volume Element for unidirectional fiber-reinforced composites: Case of a monotonic traction in its cross section. Compos. Struct. 2016, 154, 11-16. [CrossRef]

23. Riaño, L.; Belec, L.; Chailan, J.F.; Joliff, Y. Effect of interphase region on the elastic behavior of unidirectional glass-fiber/epoxy composites. Compos. Struct. 2018, 198, 109-116. [CrossRef]

24. Melro, A.R.; Camanho, P.P.; Pinho, S.T. Generation of random distribution of fibres in long-fibre reinforced composites. Compos. Sci. Technol. 2008, 68, 2092-2102. [CrossRef]

25. Ernst, G. Multiscale Analysis of textile Composites - Stiffness and Strength -. Ph.D. Thesis, Leibnitz Universität Hannover, Hannover, Germany, 2009. 
26. Massarwa, E.; Aboudi, J.; Haj-Ali, R. A multiscale progressive damage analysis for laminated composite structures using the parametric HFGMC micromechanics. Compos. Struct. 2018, 188, 159-172. [CrossRef]

27. Bai, X.; Bessa, M.A.; Melro, A.R.; Camanho, P.P.; Guo, L.; Liu, W.K. High-fidelity micromechanical modeling of the thermo-visco-plastic behavior of carbon fiber polymer matrix composites. Compos. Sci. Technol. 2015, 134, 132-141. [CrossRef]

28. Kästner, M. Skalenübergreifende Modellierung und Simulation des mechanischen Verhaltens von textilverstärktem Polypropylen unter Nutzung der XFEM. Ph.D. thesis, Technische Universität Dresden, Dresded, Germany, 2009.

29. Bowden, P.B.; Jukes, J.A. The Plastic Flow of Isotropic Polymers. J. Mater. 1972, 7, 52-63. [CrossRef]

30. Kinloch, A.J.; Young, R.J. Fracture Behaviour of Polymers, 1st ed.; Applied Science Publishers: London, UK; New York, NY, USA, 1983.

31. Haupt, P. On the mathematical modelling in continuum mechanics. Acta Mech. 1993, 100, 129-154. [CrossRef]

32. Kaddour, A.S.; Hinton, M.J. Input data for test cases used in benchmarking triaxial failure theories of composites. J. Compos. Mater. 2012, 46, 2295-2312. [CrossRef]

33. Bertram, A.; Glüge, R. Festkörpermechanik; Otto-von-Guericke-Universität Magdeburg: Magdeburg, Germany, 2013; pp. 1-295.

34. Puck, A.; Schürmann, H. Die Zug/Druck-Torsionsprüfung an rohrförmigen Probekörpern. Kunststoffe 1982, 72, 554-561.

35. Gamstedt, E.K.; Sjögren, B.A. Micromechanisms in tension-compression fatigue of composite laminates containing transverse plies. Compos. Sci. Technol. 1999, 59, 167-178. [CrossRef]

36. Ha, S.K.; Jin, K.K.; Huang, Y. Micro-Mechanics of Failure (MMF) for Continuous Fiber Reinforced Composites. J. Compos. Mater. 2008, 42, 1873-1895. [CrossRef]

37. Merodio, J.; Haughton, D.M. Bifurcation of thick-walled cylindrical shells and the mechanical response of arterial tissue affected by Marfan's syndrome. Mech. Res. Commun. 2010, 37, 1-6. [CrossRef]

38. Stassi-D'Alia, F. Flow and fracture of materials according to a new limiting condition of yelding. Meccanica 1967, 2, 175-195. [CrossRef]

39. Raghava, R.S.; Caddell, R.M.; Yeh, G.S.Y. The Macroscopic Yield Behavior of Polymers. J. Mater. Sci. 1973, 8, 225-232. [CrossRef]

40. Lapczyk, I.; Hurtado, J.A. Progressive damage modeling in fiber-reinforced materials. Compos. Part A Appl. Sci. Manuf. 2007, 38, 2333-2341. [CrossRef]

41. González, C.; LLorca, J. Mechanical behavior of unidirectional fiber-reinforced polymers under transverse compression: Microscopic mechanisms and modeling. Compos. Sci. Technol. 2007, 67, 2795-2806. [CrossRef]

42. Krause, D. Mikromechanische Untersuchung des Ermüdungsverhaltens polymerer Verbundwerkstoffe. Ph.D. Thesis, TU Braunschweig, Braunschweig, Germany, 2016. 